\title{
Activated macrophages are essential in a murine model for T cell-mediated chronic psoriasiform skin inflammation
}

\author{
Honglin Wang, ${ }^{1}$ Thorsten Peters, ${ }^{1}$ Daniel Kess, ${ }^{1}$ Anca Sindrilaru, ${ }^{1}$ Tsvetelina Oreshkova, ${ }^{1}$ \\ Nico Van Rooijen, ${ }^{2}$ Athanasios Stratis, ${ }^{3}$ Andreas C. Renkl, ${ }^{1}$ Cord Sunderkötter, ${ }^{1}$ \\ Meinhard Wlaschek, ${ }^{1}$ Ingo Haase, ${ }^{3}$ and Karin Scharffetter-Kochanek ${ }^{1}$
}

\begin{abstract}
1Department of Dermatology and Allergic Diseases, University of Ulm, Ulm, Germany. 2Department of Cell Biology, Free University, Amsterdam, The Netherlands. ${ }^{3}$ Department of Dermatology and Center for Molecular Medicine, University of Cologne (CMMC), Cologne, Germany.
\end{abstract}

\begin{abstract}
The CD18 hypomorphic (CD18hypo $) \mathrm{PL} / \mathrm{J}$ mouse model clinically resembling human psoriasis is characterized by reduced expression of the common chain of $\beta_{2}$ integrins (CD11/CD18) to only $2-16 \%$ of WT levels. Previously we found that this chronic psoriasiform skin inflammation also depends on the presence of CD4 ${ }^{+} \mathrm{T}$ cells. Herein we investigated the role of macrophages in this $C D 18^{\text {hypo }}$ mouse model. Activated macrophages were significantly increased in lesional skin as well as in inflamed skin draining lymph nodes (DLNs) of affected $C D 18^{\text {bypo }}$ mice and were identified as being an important source of TNF- $\alpha$ in vivo. Both depletion of macrophages and neutralization of TNF- $\alpha$ resulted in a significant alleviation of psoriasiform skin inflammation. As monocyte chemotactic protein 1 was enhanced in lesional skin of affected $C D 18^{b y p o}$ mice, we intradermally injected recombinant murine monocyte chemotactic protein-1 (rJE/MCP-1) alone or in combination with $r T N F-\alpha$ into the skin of healthy $C D 18^{b y p o}$ mice. Only simultaneous injection of rJE/MCP-1 and rTNF- $\alpha$, but neither substance alone, resulted in the induction of psoriasiform skin inflammation around the injection sites with recruitment and activation of macrophages. Collectively, our data suggest that maintenance of psoriasiform skin inflammation critically depends on efficient recruitment and activation of macrophages with sufficient release of TNF- $\alpha$.
\end{abstract}

\section{Introduction}

Psoriasis is regarded as a $\mathrm{T}$ cell-mediated disease $(1,2)$. However, there is an increasing body of evidence suggesting that macrophages may also be relevant for its pathophysiology (2, 3). Macrophages lining the epidermal/dermal junction have repeatedly been described in human psoriasis (3-5). In addition, neutralization of TNF- $\alpha$, a cytokine released by various cell types including macrophages, substantially improves human psoriasis (6). These anti-TNF- $\alpha$-directed agents are thought to counteract the TNF- $\alpha$ effect, thereby inhibiting crucial components of the inflammatory response (reviewed in refs. 7,8 ).

Treatment with agents targeting TNF- $\alpha$, including etanercept, a soluble p75 TNF receptor that binds to both TNF- $\alpha$ and lymphotoxin, were efficacious and safe in both psoriasis arthritis and psoriasis $(6,9)$. Etanercept is well suited for the study of the effect of blocking TNF- $\alpha$ function in the regulation of cellular events during the development of psoriasis, as its action is due to neutralization of soluble TNF- $\alpha$ and not to the depletion of cells with membrane-bound TNF- $\alpha$ (10).

Research into the pathogenesis of human psoriasis has profited at least in part from suitable animal models. Most of these, however, reveal only a single or a few aspects resembling human psoriasis (11-14). Previously, introduction of an insertion mutation

Nonstandard abbreviations used: $\mathrm{CD} 18^{\text {hypo }}, \mathrm{CD} 18$ hypomorphic; Cy3, cytochrome 3; DLN, draining lymph node; JE/MCP-1, murine monocyte chemotactic protein-1; K14, keratin 14; MHCII, MHC class II; MOMA-2, rat anti-mouse macrophages/ monocytes monoclonal antibody-2; PASI, psoriasis activity and severity index; r-, recombinant.

Conflict of interest: The authors have declared that no conflict of interest exists. Citation for this article: J. Clin. Invest. 116:2105-2114 (2006). doi:10.1172/JCI27180. in the murine CD18 gene - resulting in a duplication of exons 2 and 3 - yielded a mouse model with a severe reduction of CD18 expression, at levels only $2-16 \%$ of WT (15). Due to this CD18 hypomorphic $(C D 18$ hypo $)$ mutation, a chronic inflammatory skin disease develops in $\mathrm{PL} / \mathrm{J}$ mice that resembles human psoriasis clinically, histologically, in its polygenic nature, and in its response to therapy (16). Affected mice present with erythema, crusts, and scaling as well as abnormal keratinocyte proliferation/differentiation, subcorneal microabscesses, and an increased inflammatory infiltrate. In severely affected mice reversible alopecia was observed, a feature which may only rarely, if at all, occur in human psoriasis (17). The psoriasiform skin disease was only observed when the CD18hypo mutation was backcrossed on the PL/J, but not on the C57BL/ 6 or $129 \mathrm{~Sv}$, inbred mouse strains. Homozygous mutant mice on a PL/J $\times$ C57BL/6 F1 background did not develop the disease, despite the $C D 18$ hypo mutation. Backcross analysis suggests that, in addition to the $C D 18^{\text {hypo }}$ mutation, a small number of other genes determine susceptibility to the disease (16). Hence, the CD18ypo PL/J model qualifies as a polygenic model for chronic skin inflammation. Such a polygenic nature has been claimed for human psoriasis (18).

As in patients treated for severe psoriasis $(19,20)$, this chronic psoriasiform skin disease of the CD18 hypo $\mathrm{PL} / \mathrm{J}$ mouse model can be suppressed by corticosteroids (dexamethasone), suggesting the involvement of an inflammatory process. T cells are important in the generation of the inflammatory skin disease in this CD18bypo $\mathrm{PL} / \mathrm{J}$ model (21). This is analogous to affected skin of psoriasis patients in which $\mathrm{CD}^{+} \mathrm{T}$ cells prevail (22).

CD18 represents the common $\beta_{2}$ chain of the $\beta_{2}$ integrin family. These integrins (CD11/CD18) are leukocyte adhesion molecules exclusively expressed on hemopoietic cells and are responsible for 

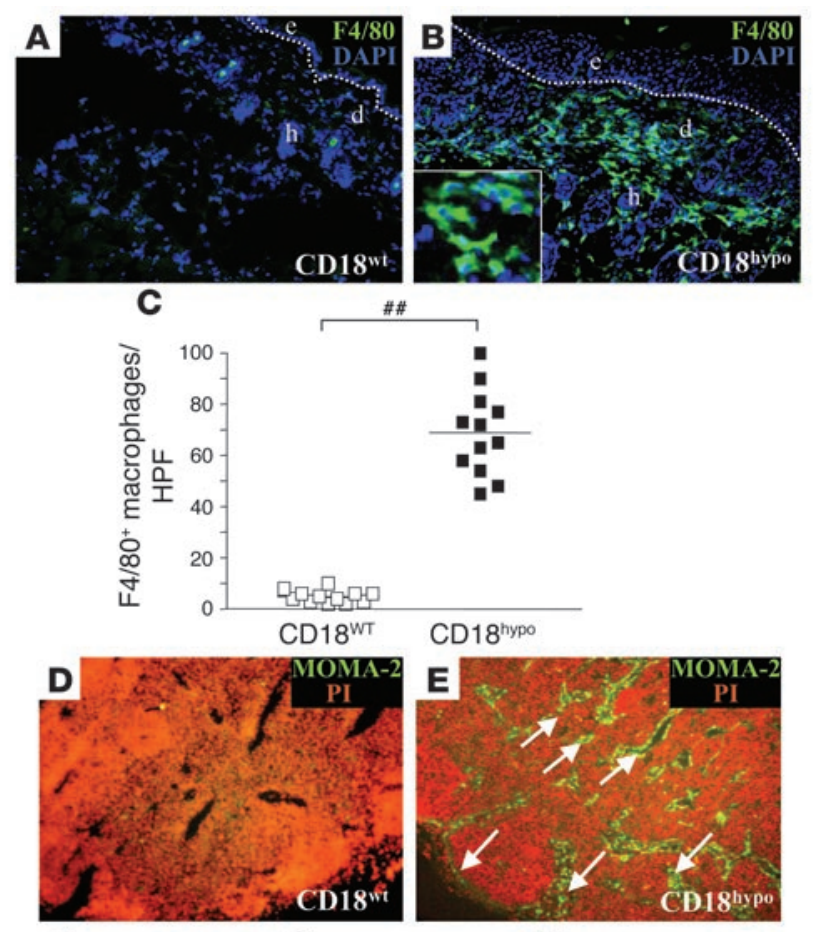

$\mathbf{F}$ G
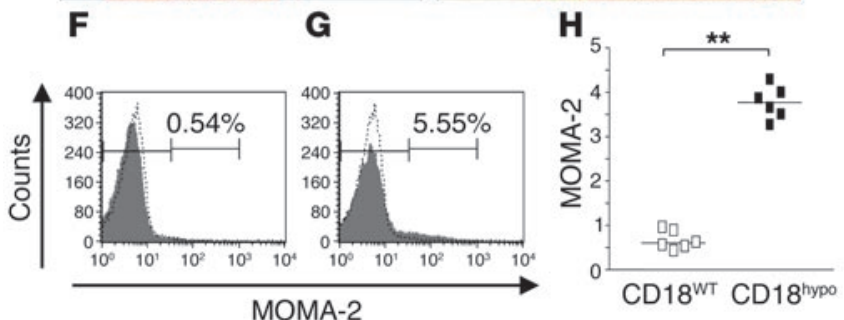

cell-cell contacts in a variety of inflammatory interactions (23). At present, 4 different $\beta_{2}$ integrins have been characterized, all of which are heterodimeric cell-surface molecules consisting of the CD18 molecule and one of the CD11 molecules: CD11a, -b, -c, or -d. These heterodimeric molecules interact with more than 20 known ligands, the most prominent of which belong to the ICAM family (24).

The exact nature of reduced expression levels of CD18 in the spontaneous manifestation of the chronic inflammatory skin disease in the CD18 hypo PL/J model is not completely understood. However, the CD18-dependent extravasation of T cells is mandatory. Interestingly, this chronic inflammatory skin disease develops only in the CD18 hypo PL/J model with some expression of CD18, not in the CD18 $8^{\text {null }} \mathrm{PL} / \mathrm{J}$ mutants with no expression of CD18 (21, 25). Among several possibilities, reduced CD18 expression may cause disturbances in the formation of the immunological synapse resulting in the generation and persistence of autoreactive cells, e.g., by abnormal deletion in the thymus. In fact, $\beta_{2}$ integrins are involved in thymic T cell development and selection $(26,27)$.

The pathogenic role of $\beta_{2}$ integrins in human psoriasis and other inflammatory skin diseases is even less well understood. Circumstantial evidence indicating that reduced CD18 expression may causally be involved in the development of this psoriasiform dermatitis comes from the clinical observation that some patients suffering from leukocyte adhesion deficiency syndrome- 1 with only moderately reduced CD18 expression levels develop a psoriasiform skin disease (28). Linkage analysis

\section{Figure 1}

Increase in macrophages numbers in lesional skin and skin DLNs of affected CD18 ${ }^{\text {hypo }}$ mice. (A and B) Skin cryosections from CD18 ${ }^{W T}$ (A) and affected $C D 18^{\text {hypo }}$ mice (B) were stained with F4/80-Alexa 488 for infiltrating macrophages (green) into the skin. Cell nuclei (blue) were counterstained with DAPI. e, epidermis; d, dermis; h, hair follicle. Dotted lines indicate the border between epidermis and dermis. Original magnification, $\times 40$; inset, $\times 100$. (C) To quantify macrophages in the skin of affected $C D 18^{\text {hypo }}$ and $C D 18^{W T}$ mice, the positively stained cells were calculated. For all measurements, the median of macrophages counted in 12 high-power fields (HPF) is presented $(n=4)$. $\# P<0.0001$, Student's $t$ test. (D and E) Immunostaining with macrophage/monocyte-FITC (clone MOMA-2) was performed on cryosections of skin DLNs from CD18 ${ }^{W T}$ (D) and affected $C D 18^{\text {hypo }}$ mice (E). Infiltrated macrophages (green) were found in the medullar and subcapsular sinuses, as indicated by arrows. Cell nuclei (red) were counterstained with propidium iodide $(\mathrm{PI})$. Original magnification, $\times 20$. ( $\mathbf{F}$ and $\mathbf{G})$ To quantify macrophages in the skin DLNs of $C D 18^{W T}(\mathbf{F})$ and affected $C D 18^{\text {hypo }}$ mice $(\mathbf{G})$, skin DLN cells were labeled with MOMA-2FITC and analyzed by flow cytometry. Dotted line, isotype control; gray histogram, MOMA-2 staining. (H) Total number of macrophages

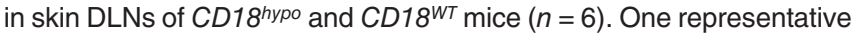
experiment of 3 is shown. ${ }^{* *} P<0.01$, Student's $t$ test.

of psoriasis families has identified a region on chromosome 17, including - among other loci - the ICAM-2 locus, an important ligand of the CD11/CD18 heterodimers (29). Furthermore, polymorphisms in the CD18 gene have been found that predispose to autoimmune diseases $(30,31)$.

As CD18 also is an important membrane receptor/molecule for macrophages, and as macrophages are increased in number in human psoriasis and may play a role in the full development of psoriasis, we decided to investigate their involvement in the CD18 hypo PL/J model for chronic inflammatory skin disease.

In order to gain more insight into the role of macrophages in various models of inflammation, several approaches have been used to reduce macrophage activity, including blockade of secreted cytokines (32), and downregulation of macrophage activity (33) using Ricin A-coupled antibodies directed against the high-affinity IgG receptor CD64 (34) or liposomes containing dichloromethylene diphosphonate (clodronate liposomes) (35). Clodronate liposomes have been shown to efficiently and selectively deplete phagocytic macrophages by apoptosis from spleen, liver, lymph nodes, and skin, while other nonphagocytic immunocompetent cells such as T cells, B cells, and dendritic cells are not eliminated (36). In this regard it is important for our studies that intradermal injections of clodronate liposomes did not deplete mature Langerhans cells from the epidermis (35).

Here we show that the number of activated macrophages was greatly increased in the lesional skin of the chronic inflammatory skin disease in $C D 18^{\text {hypo }}$ mice, corresponding to human psoriasis. In addition, activated macrophages were identified as an important source of TNF- $\alpha$ in the lesional skin of CD18 bypo mice. Interestingly, the depletion of macrophages in the skin or skin draining lymph nodes (DLNs) through the administration of clodronate liposomes, when coupled with the neutralization of TNF- $\alpha$ function by etanercept, as in human psoriasis studies $(6,9)$, resulted in a significant reduction of the severity of the chronic psoriasiform skin inflammation. In addition, we show that the mere attraction or partial activation of macrophages into the skin by recombinant murine monocyte chemotactic protein-1 (rJE/MCP-1) or LPS was not sufficient to trigger the chronic psoriasiform inflammatory skin disease. 

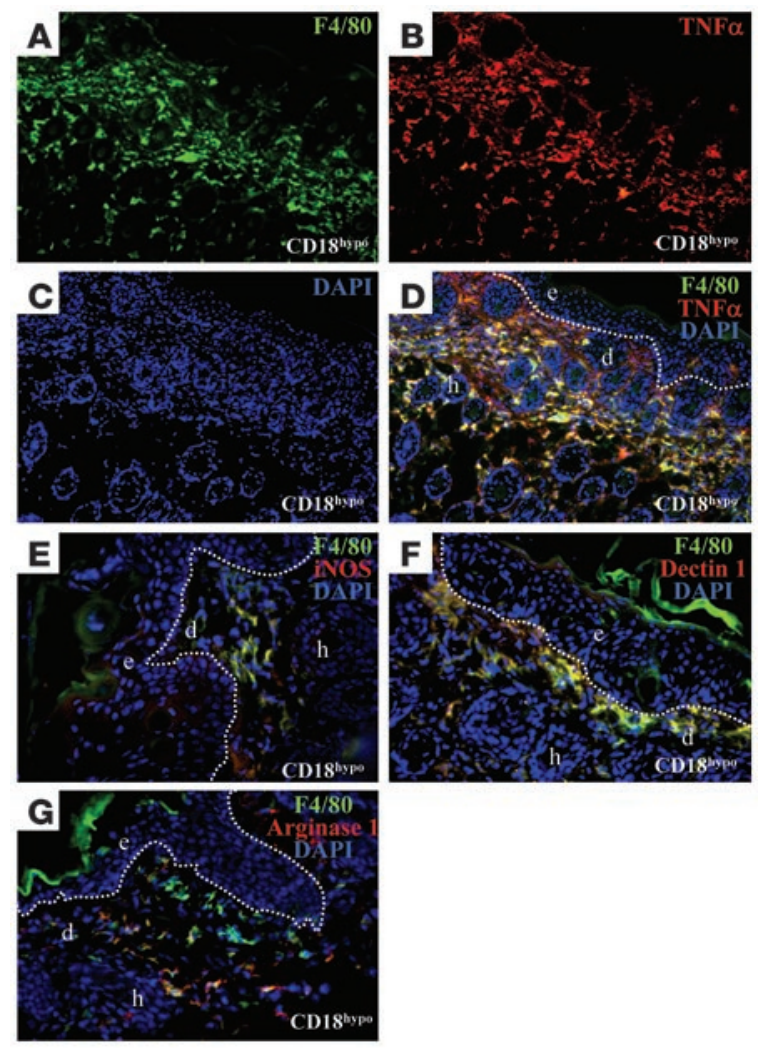

In contrast, combined injection of the macrophage-recruiting rJE/MCP-1 and the macrophage-activating rTNF- $\alpha$ resulted in the development of the chronic psoriasiform skin inflammation due to the activation of macrophages in healthy skin of CD18hypo $\mathrm{PL} / \mathrm{J}$ mice. Our data convincingly show that depletion of activated macrophages results in the resolution of the chronic psoriasiform skin inflammation, demonstrating for the first time to our knowledge an essential role of macrophages in the pathogenesis of a $\mathrm{T}$ cell-mediated murine model for chronic inflammatory skin disease that shares many aspects with human psoriasis.

\section{Results}

Macrophages are markedly increased in lesional skin as well as in DLNs of inflamed skin in affected CD18 bypo mice. Increased numbers of macrophages are found in the dermis of lesional skin in human psoriasis (3-5). To investigate the composition of the inflammatory cellular infiltrate in the psoriasiform skin and DLNs of CD18hypo mice, 12 skin sections taken from $C D 18^{\text {hypo }}$ and $C D 18^{W T}$ mice $(n=4)$ were immunostained with F4/80 to detect mature tissue macrophages and FITC-conjugated rat anti-mouse macrophages/monocytes monoclonal antibody-2 (MOMA-2; Serotec) specific for the less mature monocyte/macrophage population in lymph nodes. Compared with $C D 18^{W T}$ skin, with only few macrophages in the dermis (Figure 1A), the number of macrophages was significantly increased in the dermis of lesional skin in affected CD18 $8^{\text {hypo }}$ mice $(P<0.0001$; Figure 1, B and C). In skin DLNs of WT mice almost no macrophages were observed (Figure 1D), whereas many macrophages - which had apparently entered via the medullar and subcapsular sinuses of skin DLNs - were found in affected CD18 bypo mice (Figure 1E, arrows). To better quantify and characterize macrophages in skin DLNs, we performed flow cytometric analysis

\section{Figure 2}

Activated macrophages are an important source of TNF- $\alpha$ in the lesional skin of affected $C D 18^{\text {hypo }}$ mice. Double immunostaining with anti-mouse TNF- $\alpha$ and F4/80 mAb was performed on cryosections

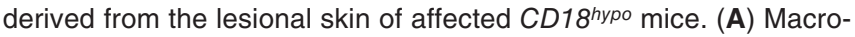
phages (green) stained with F4/80-Alexa 488. (B) TNF- $\alpha$ (red) stained with TNF- $\alpha-$-Cy3. (C) Cell nuclei were stained with DAPI (blue). (D) Overlay depicting double staining of TNF- $\alpha$ and macrophages (yellow). (D-G) Both classically and alternatively activated macrophages were present in the lesional skin of $C D 18^{\text {hypo }}$ mice. To characterize the activation pattern of macrophages infiltrating the skin of affected CD18 hypo mice, cryosections from lesional skin were double stained with F4/80-Alexa 488 (green) and the markers of classically activated macrophages TNF- $\alpha-$ Cy3 (red) (D) and iNOS-Cy3 (red) (E) or with the markers of alternatively activated macrophages Dectin 1-Cy3 (red) (F) and Arginase 1-Сy3 (G). Overlay (yellow) represents double-positive cells, i.e., macrophages bearing specific activation markers. Cell nuclei were counterstained with DAPI (blue). Dotted lines indicate the border between epidermis and dermis. Original magnification, $\times 20$.

for macrophages labeled with MOMA-2 $(n=6)$. In contrast to WT mice (Figure $1 \mathrm{~F}$ ), the number of MOMA-2+ macrophages was significantly higher in inflamed skin DLNs of affected CD18 hypo mice $(P<0.01$; Figure 1, G and $\mathrm{H})$.

Macrophages are an important source of TNF- $\alpha$ in the lesional skin of affected CD18hypo mice. To investigate whether activated macrophages are an important source of TNF- $\alpha$ in the lesional skin of affected $C D 18^{\text {hypo }}$ mice, double immunofluorescence staining was performed on cryosections derived from the lesional skin of affected CD18 hypo mice with TNF- $\alpha$ and F4/80 mAbs. As expected, positive macrophage staining with F4/80 was found in lesional skin of CD18 ${ }^{\text {hypo }}$ mice (Figure 2A). An identical staining pattern was found using TNF- $\alpha$ mAb (Figure 2B). DAPI staining was performed to identify all nuclei (Figure 2C). Staining with TNF- $\alpha, F 4 / 80$, and DAPI clearly shows that almost all macrophages produced TNF- $\alpha$ in the lesional skin of affected CD18 bypo mice (Figure 2D). Interestingly, both classically activated macrophages, characterized by the production of TNF- $\alpha$ (Figure 2D) and iNOS (Figure 2E), and alternatively activated macrophages, characterized by enhanced Dectin 1 (Figure 2F) and Arginase 1 (Figure 2G) expression, were present in the lesional skin of CD18 hypo mice. In addition to a high number of macrophages, infiltrating mast cells and endothelial cells were also sources of TNF- $\alpha$ in the lesional skin of CD18 ${ }^{\text {hypo }}$ mice (Supplemental Figure 2; supplemental material available online with this article; doi:10.1172/JCI27180DS1).

Blocking TNF- $\alpha$ by etanercept results in improvement of the psoriasiform skin inflammation and is accompanied by reduced numbers of macrophages and decreased expression of MHC class II molecules and TNF- $\alpha$. TNF- $\alpha$ has been shown to play a central role in the cytokine network of human psoriasis (8), and TNF- $\alpha$-inhibitory agents, including etanercept, have been successfully used in the treatment of human psoriasis $(6,9)$. Based on our observation that macrophages are a major source for TNF- $\alpha$, we analyzed its relevance in the formation of the psoriasiform skin disease in our mouse model. We neutralized TNF- $\alpha$ by administrating etanercept once a day at a dose of $100 \mu \mathrm{g}$ per mouse. We found that this protocol significantly decreased the adapted psoriasis activity and severity index (PASI) score after 30 days of treatment $(6 \pm 0.71$ versus $2.4 \pm 0.55 ; P=0.0079$; Figure $3 \mathrm{~A})$. In contrast, no significant improvement was observed in the mice treated with $0.9 \% \mathrm{NaCl}$ 

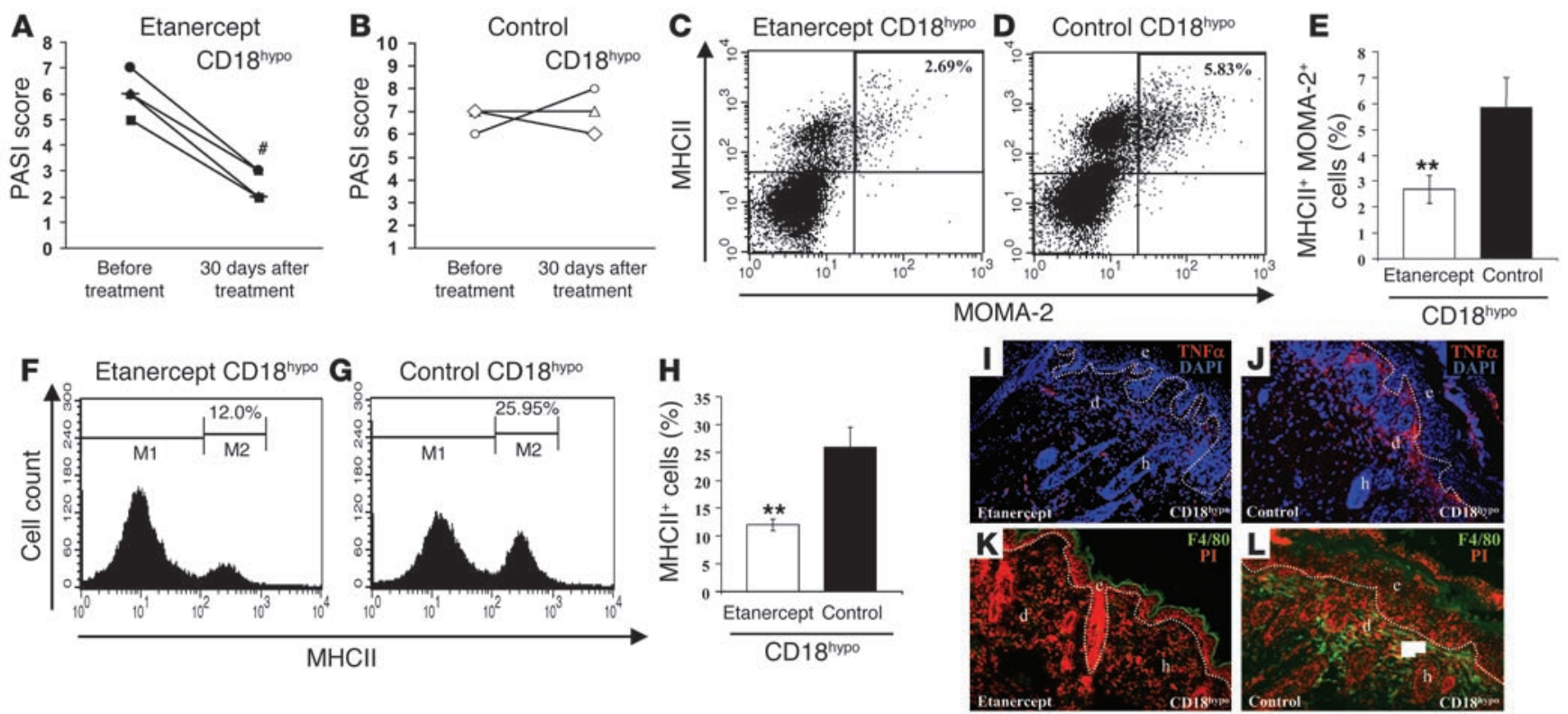

Figure 3

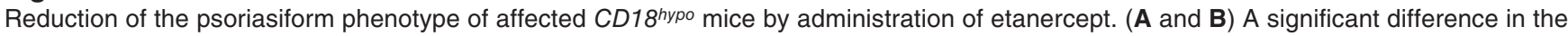
adapted PASI score appeared after treatment with etanercept $\left(\mathbf{A} ; n=5 ;{ }^{\#} P=0.0079\right.$, Student's $t$ test), but not after treatment with $0.9 \% \mathrm{NaCl}$ control (B; $n=3 ; P=0.6514$, Student's $t$ test). (C-E) Significantly reduced numbers of macrophages in skin DLNs of affected $C D 18^{\text {hypo }}$ mice treated with etanercept $(\mathbf{C})$ were observed after 30 days compared with controls $(\mathbf{D}$ and $\mathbf{E})$. $(\mathbf{F}-\mathbf{H})$ Decreased numbers of $\mathbf{M H C l l}^{+}$cells in skin DLNs of affected $C D 18^{\text {hypo }}$ mice treated with etanercept $(\mathbf{F})$ were detected after 30 days compared with controls $(\mathbf{G}$ and $\mathbf{H})$. $\mathbf{M}_{1}, \mathbf{M H C I I}^{-}$cells; $\mathrm{M}_{2}$, MHCll+ cells. ${ }^{* *} P<0.01$, Student's $t$ test. (I and J) Decreased expression of TNF- $\alpha$ (red) was observed in the skin of affected CD18 ${ }^{\text {hypo }}$ mice treated with etanercept after 30 days (I) compared with controls (J). Cell nuclei were stained with DAPI (blue). (K and L) Reduced numbers of $\mathrm{F} 4 / 80^{+}$macrophages (green) were observed in the skin of affected $C D 18^{\text {hypo }}$ mice treated with etanercept after 30 days (K) compared with controls (L). Cell nuclei (red) were counterstained with propidium iodide. Original magnification, $\times 20$.

(i.e., controls; $P=0.6514$; Figure 3B). Interestingly, neutralization of TNF- $\alpha$ did not only reduce the numbers of MHC class IIand MOMA-2-positive (MHCII+ ${ }^{+} O \mathrm{MA}^{2}{ }^{+}$) macrophages (Figure 3, C-E), but also the numbers of $\mathrm{MHCII}^{+}$cells in inflamed skin DLNs of CD18 bypo mice (Figure 3, F-H). Furthermore, neutralization of TNF- $\alpha$ also decreased expression of TNF- $\alpha$ in lesional skin of $C D 18^{\text {hypo }}$ mice (Figure 3I), while TNF- $\alpha$ was broadly expressed in $C D 18^{\text {bypo }}$ mice injected with $\mathrm{NaCl}$ (Figure 3J). In addition, the numbers of $\mathrm{F} 4 / 80^{+}$macrophages decreased in the dermis of etanercept-treated CD18 hypo mice (Figure $3 \mathrm{~K}$ ) compared with affected CD18 ${ }^{\text {hypo }}$ controls (Figure $3 \mathrm{~L}$ ).

Improvement of the chronic psoriasiform skin inflammation, with concomitant decrease in TNF- $\alpha$ expression, after depletion of skin macrophages. In order to analyze whether activated macrophages are mandatory in the pathogenesis of the psoriasiform skin inflammation in CD18 ${ }^{\text {hypo }}$ mice, we eliminated macrophages from skin of affected $C D 18$ bypo mice using clodronate liposomes. The specificity of clodronate liposomes for the depletion of phagocytic cells has repeatedly been shown (37-39). We furthermore found that clodronate liposomes specifically depleted macrophages from the skin 24 hours after injection, but had no significant effect on T cells, neutrophils, mast cells, or mature Langerhans/ dendritic cells (Supplemental Figure 1). Depletion of macrophages in $C D 18^{\text {hypo }}$ mice with a severe inflammatory phenotype (Figure 4A) led to a remarkable improvement of the psoriasiform skin inflammation after 6 weeks of treatment (Figure 4B). To evaluate the severity of the psoriasiform phenotype, an adapted PASI score (21) was used for affected CD18 hypo mice before and after treatment with clodronate liposomes or control liposomes. For $C D 18^{\text {hypo }}$ mice, PASI was scored accordingly: 0, no symptoms; 1 , slight erythema of the ears; 2 , strong erythema of the ears; 3 , slight hair loss on the head; 4, extensive hair loss including the trunk; 5 , slight hair loss, isolated scaling; 6, extensive hair loss, isolated scaling; 7 , extensive hair loss, widespread slight scaling; 8 , moderate scaling of a large area of the body; 9 , widespread hair loss, strong scaling of few smaller areas; 10, extensive hair loss, extensive scaling of a large area of the body. The reduction in severity and extent of erythema, plaque formation, and scaling after treatment with clodronate liposomes was highly significant ( $7.67 \pm 1.03$ versus $2.33 \pm 0.52 ; P=0.0022$; Figure $4 \mathrm{C}$ ). In contrast, no significant changes in PASI score were observed in 4 mice treated with control liposomes $(P=0.3429$; Figure 4D).

Administration of clodronate liposomes resulted in an almost complete removal of macrophages from the injected skin areas (Figure 4F) as well as from skin DLNs (Figure 4H), in contrast to the treatment with control liposomes (Figure 4, E and G).

Immunohistochemical staining for TNF- $\alpha$ on cryosections of skin from affected $C D 18^{\text {hypo }}$ mice treated with control liposomes showed strong TNF- $\alpha$ expression in affected skin (Figure 4I), while in skin treated with clodronate liposomes, TNF- $\alpha$ expression was virtually undetectable (Figure 4J). Importantly, immunostaining for GR-1+ 40 days after clodronate treatment of diseased $C D 18^{\text {hypo }} \mathrm{PL} / \mathrm{J}$ mice - a time point at which skin inflammation and phenotype had completely resolved - revealed almost no GR-1 $1^{+}$granulocytes in clodronate-treated mice ( $7 \pm 3.8$ polymorphonuclear granulocytes per high-power field; 

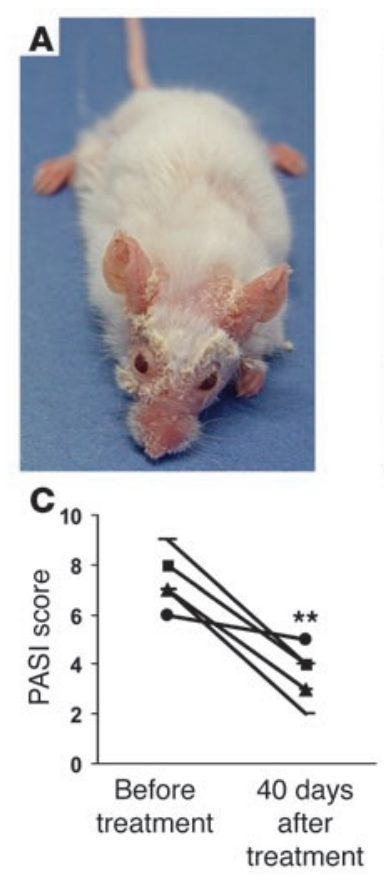
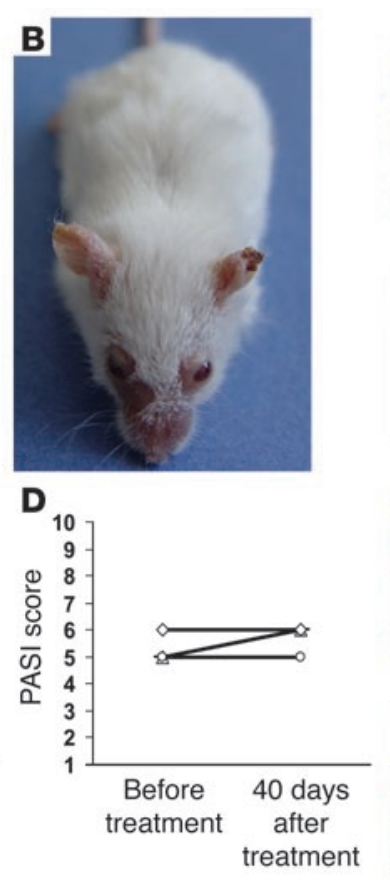
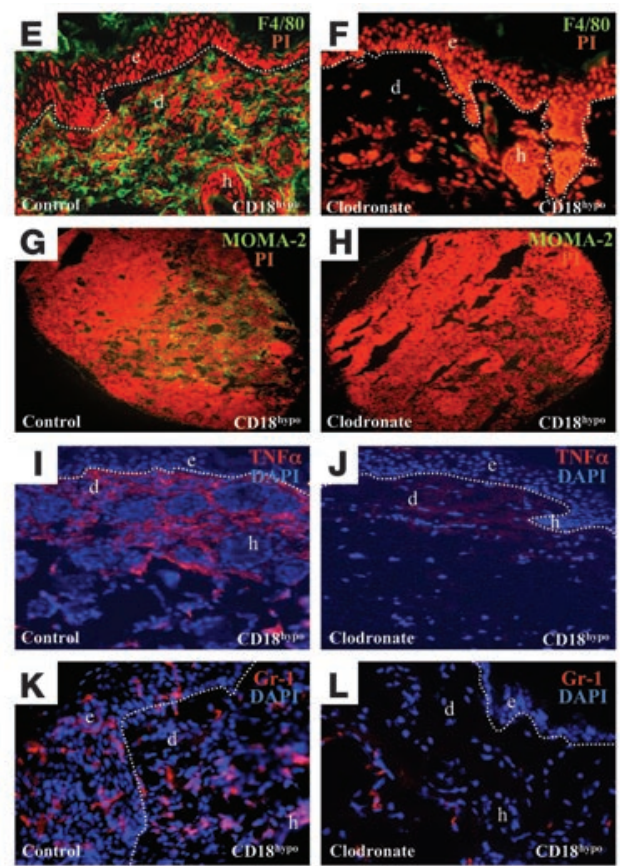

Figure 4

Improvement of the psoriasiform phenotype of affected $C D 18^{\text {hypo }}$ mice by depletion of macrophages after local injection with clodronate liposomes. Clodronate liposomes were injected subcutaneously $(50 \mathrm{mg} / 200 \mu \mathrm{l}$, administered weekly). (A and B) Representative clinical picture of a CD18 hypo mouse with severe psoriasiform dermatitis before $(\mathbf{A})$ and after 40 days of treatment with clodronate liposomes (B). (C and $\mathbf{D})$ The severity of the psoriasiform phenotype was significantly reduced after clodronate treatment $(\mathbf{C} ; n=6)$ but not after treatment with control liposomes $(\mathbf{D} ; n=4$; $P=0.3429$, Student's $t$ test). ${ }^{* *} P<0.01$. (E-H) Efficiency of depletion was evaluated by immunostaining. Clodronate liposome treatment dramatically reduced the number of $\mathrm{F} 4 / 80^{+}$macrophages (green) in injected skin areas $(\mathrm{F})$ and skin $\mathrm{DLNs}(\mathrm{H})$ of $C D 18^{\text {hypo }}$ mice compared with skin $(\mathrm{E})$ and skin DLNs (G) from mice treated with control liposomes. Depletion of inflammatory macrophages also caused a decrease in TNF- $\alpha$ expression (red): skin samples from CD18 hypo mice treated with control liposomes showed strong TNF- $\alpha$ expression (I), whereas expression in clodronate liposome-treated skin was virtually undetectable (J). GR-1 staining (red) of skin from CD18 hypo mice 40 days after clodronate liposome injection (K), when skin inflammation and phenotype had completely resolved, revealed that granulocytes were removed, whereas GR-1+ granulocytes were still present after PBS-liposome treatment $(\mathbf{L})$. Dotted lines indicate the border between epidermis and dermis. Cell nuclei were counterstained with propidium iodine (red) or DAPI (blue) as indicated. Original magnification, $\times 40(\mathbf{E}, \mathbf{F}, \mathbf{K}$, and $\mathbf{L}) ; \times 10(\mathbf{G}$ and $\mathbf{H})$.

Figure 4L) compared with the severely affected PBS-treated mice (63 \pm 17.8 polymorphonuclear granulocytes per high-power field; $P<0.0001$; Figure $4 \mathrm{~K}$ ). This immunostaining provided further measure of successful resolution of chronic inflammation after clodronate liposome injections.

Both MCP-1 mRNA and protein levels are significantly enhanced in psoriatic lesions of CD18hypo mice. As MCP-1 is an important chemokine for the recruitment of monocytes/macrophages (40), we addressed the question of whether MCP-1 expression correlates with the observed macrophage infiltration in lesional skin of $C D 18^{\text {hypo }}$ mice. Three samples derived from lesional skin of affected $C D 18^{\text {bypo }}$ mice were pooled to perform a semiquantitative realtime RT-PCR. Lesional skin from the psoriasis mouse model presented higher levels of MCP-1 mRNA compared with CD18WT (Figure 5A). To further characterize this difference, we performed a quantitative real-time RT-PCR, which confirmed that MCP-1 expression was significantly increased in lesional skin of affected $C D 18^{\text {hypo }}$ mice compared with healthy $C D 18^{W T}$ mice $(n=3 ; P<0.05$; Figure 5B). Additionally, ELISA of skin samples demonstrated that on the protein level the concentration of MCP-1 was substantially higher in lesional skin of affected CD18 bypo mice that of CD18WT mice (1,061.8 versus $251.9 \mathrm{pg} / \mathrm{mg}$ tissue; $P<0.01$; Figure $5 \mathrm{C}$ ).
Simultaneous injection of $r J E / M C P-1$ and $r T N F-\alpha$ results in the induction of the psoriasiform skin inflammation in healthy skin of CD18bypo $P L / J$ mice. Administration of murine rJE/MCP-1 recruited but did not activate macrophages, while LPS recruited and only transiently activated macrophages with only low and intermittent production of TNF- $\alpha$ (Supplemental Results and Supplemental Figures 3 and 4). Injection of rTNF- $\alpha$ alone did not recruit macrophages and T cells (data not shown). Neither administration of rJE/MCP-1 nor LPS nor $r$ TNF- $\alpha$ alone resulted in the induction of chronic psoriasiform skin inflammation. In order to determine whether the synergistic action of a factor recruiting macrophages and a macrophage-activating proinflammatory cytokine results in the induction of the psoriasiform skin inflammation, we simultaneously injected rJE/ MCP-1 and rTNF- $\alpha$. Indeed, 4 days after combined administration of rJE/MCP-1 and rTNF- $\alpha$ in unaffected skin of CD18hypo mice, we were able to induce skin lesions around the injection site identical to those seen in the spontaneously occurring chronic psoriasiform skin inflammation in CD18 bypo PL/J mice (Figure 6, A-F). Interestingly, the clinical manifestation of the chronic psoriasiform skin inflammation was reflected histologically and by immunostaining showing accumulation and activation of macrophages (Figure 6, $\mathrm{G}$ and $\mathrm{H})$. However, virtually no recruitment of $\mathrm{CD}^{+} \mathrm{T}$ cells was observed at day 10 after treatment (Figure 6, I and J). 
A

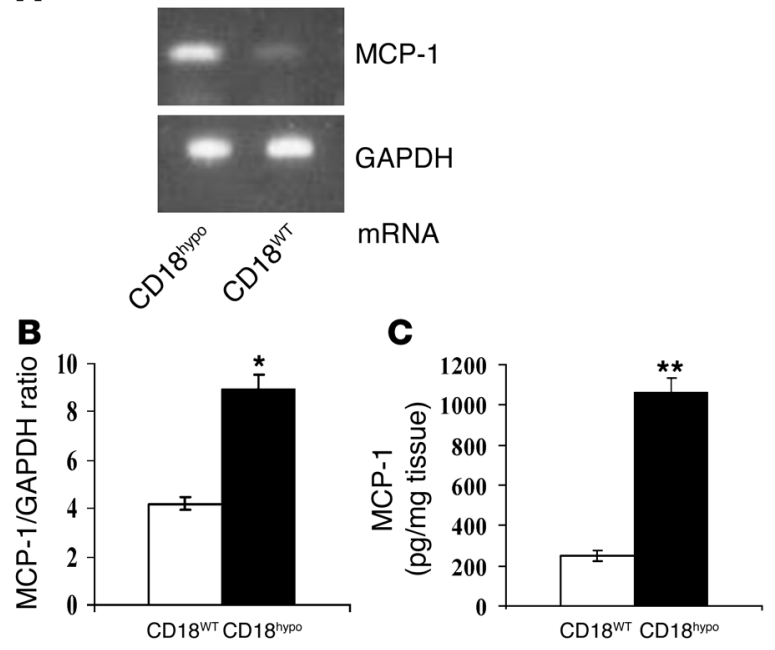

\section{Discussion}

Human psoriasis is regarded as a T cell-mediated disease (7). Previously we demonstrated that $\mathrm{CD} 4^{+} \mathrm{T}$ cells are crucial for the generation of the psoriasiform skin disease in the $\mathrm{PL} / \mathrm{J} C D 18^{\text {hypo }}$ psoriasis mouse model (21). In our current study we observed an increased number of macrophages directly beneath the epidermis of lesional skin of $C D 18^{\text {hypo }}$ mice compared with $C D 18^{W T}$ mice. Interestingly, the infiltrating macrophages presented both classical and alternative activation markers, as similarly described for human psoriasis (41). The pathogenic involvement of these subepidermally located macrophages, designated epithelium-lining macrophages, is largely unknown in human psoriasis $(2-5,42)$. Therefore, we used the PL/J CD18 ${ }^{\text {hypo }}$ mouse model herein to study a potential role of macrophages in the pathogenesis of this chronic psoriasiform skin inflammation. By means of macrophage depletion and neutralization of TNF- $\alpha$ we show that activated, TNF- $\alpha$-releasing macrophages are causally involved in the pathogenesis of this psoriasiform skin inflammation. Both procedures resulted in a decrease of the adapted PASI score, indicating improvement or resolution of the chronic psoriasiform skin inflammation.

Since MCP-1 expression is high at the epidermal/dermal junction in human plaque psoriasis and MCP-1 has strong macrophagerecruiting properties $(3,40)$, we next investigated MCP-1 expression and its function in our mouse model in more detail. Both MCP-1 mRNA and protein levels were considerably enhanced in lesional skin of affected $C D 18^{\text {hypo }}$ mice compared with $C D 18^{W T}$ mice. This may explain the strong infiltration by macrophages of the dermis in lesional skin of affected $C D 18^{\text {hypo }}$ mice. A recent report further indicated that MCP-1 binds to the chemokine receptor CCR2, which was demonstrated to be expressed on peripheral blood monocytes from patients with psoriasis (42) and also on a subtype of murine monocytes principally involved in inflammation (38). Thus interaction of MCP- 1 and CCR2 is likely to be of importance for monocyte/ macrophage trafficking in inflammatory skin disorders (43).

After intradermal injection of rJE/MCP-1, we in fact observed a distinct infiltration of monocytes/macrophages in the dermis of healthy CD18hypo mice. However, rJE/MCP-1-recruited macrophages failed to trigger psoriasiform skin disease in healthy CD1 $8^{\text {hypo }}$ mice even after 8 weeks of observation. Our data is in line with MCP-1 transgenic mice, in which MCP-1 overexpression led

\section{Figure 5}

Enhanced MCP-1 mRNA and protein levels in the lesional skin of affected $C D 18^{\text {hypo }}$ mice. Total RNA was extracted from skin of affected $C D 18^{\text {hypo }}$ and $C D 18^{W T}$ mice $(n=3)$. CDNA was analyzed for MCP-1 and GAPDH mRNA expression by semiquantitative RT-PCR. (A) Specific bands for MCP-1 (142 bp) and internal control GAPDH were visualized by ethidium bromide in $1 \%$ agarose gels. (B and $\mathbf{C}$ ) Both MCP-1 mRNA levels as determined by quantitative real-time RT-PCR (B) and MCP-1 protein levels as determined by specific MCP-1 ELISA (C) were increased in lesional skin of affected CD18hypo mice compared with $C D 18^{W T}$ controls. MCP-1 protein levels are expressed in relation to tissue weight as the mean \pm SD for triplicate samples of each mouse. ${ }^{*} P<0.05 ;{ }^{*} P<0.01$.

to a marked increase in monocytes/macrophages in lung and skin but did not cause clinically relevant inflammatory phenotypes (44, 45). Apart from rJE/MCP-1, neither LPS nor rTNF- $\alpha$ alone were able to elicit the psoriasiform skin disease. In contrast, the combination of rJE/MCP-1 and rTNF- $\alpha$ induced the inflammatory skin disease. These data suggest that recruitment and sustained activation of macrophages are required for perpetuated TNF- $\alpha$ release with subsequent development of the chronic psoriasiform inflammation. Interestingly, after rJE/MCP- 1 and rTNF- $\alpha$ administration we did not observe $\mathrm{CD} 4^{+} \mathrm{T}$ cells in the injected area. Even though $\mathrm{CD} 4^{+} \mathrm{T}$ cells have earlier been shown to activate macrophages by enhanced IFN- $\gamma$ release (46), our data suggest that once macrophages are activated directly by proinflammatory cytokines such as TNF- $\alpha$, they have the capacity to induce the psoriasiform inflammatory skin disease independently of $\mathrm{CD}^{+} \mathrm{T}$ cells. TNF- $\alpha$ was recognized as an important cytokine in the pathogenesis of psoriasis as early as 1991 (8). The suggested cellular sources of TNF- $\alpha$ release included $\mathrm{T}$ lymphocytes, mast cells, and endothelial cells $(47,48)$. We found that macrophages represent a very important source of TNF- $\alpha$. Interestingly, previous in vitro data showed that TNF- $\alpha$ perpetuates macrophage activation via increased TNF- $\alpha$ production by macrophages themselves (49). This finding was confirmed in vivo in our model. Hence our data provide, at least in part, a mechanistic basis by which activated, TNF- $\alpha$-releasing macrophages play an essential role in this chronic psoriasiform skin disease. This may provide an insight into the successful therapy of human psoriasis with a variety of TNF- $\alpha$-inhibitory agents.

Apart from the success of directly targeting TNF- $\alpha$ in the treatment of human psoriasis $(6,9)$, therapies that most likely impair macrophage function by diminishing the production and release of TNF- $\alpha$, such as IL-4 (50), IL-11 (51), and anti-IL-12 (52), have previously been reported to improve this medical condition. Thus, in addition to other beneficial effects, these agents may also exert their therapeutic efficacy by silencing macrophages. Due to the impressive success of anti-TNF- $\alpha$ therapies, the next generation of small molecules targeting different steps of TNF- $\alpha$ signaling have been developed, some of which are currently being tested in clinical trials (53).

As subcorneal microabscesses consisting of neutrophils and neutrophil infiltration of the dermis are histological features both in human skin psoriasis (54) and in the PL/J CD18 bypo model (16), we set out to more precisely address their role in our model of chronic psoriasiform skin inflammation. Our experiments show that depletion of GR-1 $1^{+}$neutrophils using neutralizing antibodies, as confirmed by FACS analysis and immunostaining of the skin, did not result in a decrease in the adapted PASI score, reflecting 

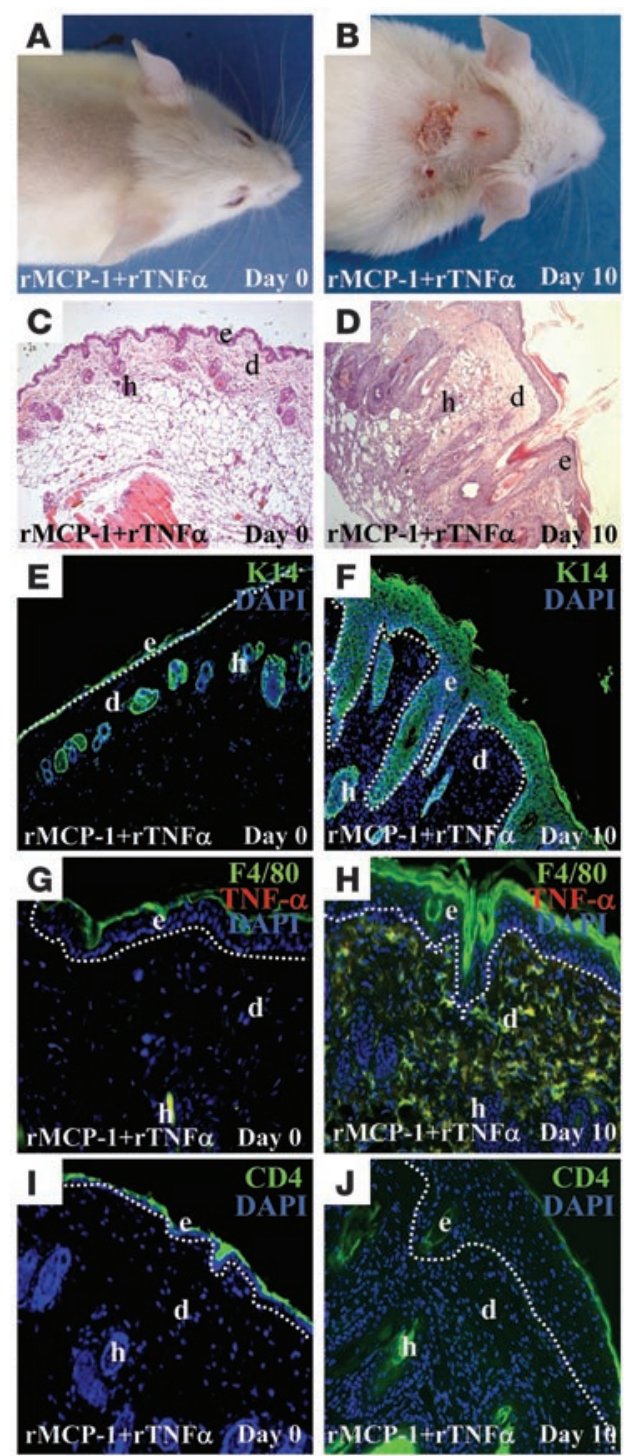

the severity of the skin inflammation (Supplemental Figure 5). This, together with new data by Stratis et al. (55), strongly suggests that the role of neutrophils is not causal for the maintenance of the skin inflammation, while macrophages do play a causal role in both models for psoriasiform skin inflammation. This is particularly interesting, as it hints to a so far unknown multifaceted role of macrophages in different inflammatory processes. We herein showed the mandatory requirement for macrophages in the $\mathrm{T}$ cell-mediated $C D 18^{\text {hypo }}$ model for chronic psoriasiform skin inflammation, while Stratis and coauthors convincingly showed that repression of the NF- $\mathrm{kB}$ pathway in keratinocytes drives a $\mathrm{T}$ cell-independent but macrophage-induced development of a psoriasis-like skin disease (55). In our model we previously found that depletion of $\mathrm{CD}^{+} \mathrm{T}$ cells using neutralizing antibodies resulted in complete resolution of the psoriasiform skin inflammation (21), strongly suggesting that $\mathrm{CD} 4^{+} \mathrm{T}$ cells play a critical role in the pathogenesis of this disease.

In addition, the psoriasiform inflammatory skin disease did not develop in the PL/J CD18 $8^{\text {null }}$ mice, which have no CD18 expression and in which macrophages are able to emigrate into the skin but

\section{Figure 6}

The injection of a combination of rJE/MCP-1 and rTNF- $\alpha$ induces psoriasiform inflammatory skin lesions in CD18 hypo PL/J mice. Neither injection of rJE/MCP-1 nor rTNF- $\alpha$ alone resulted in psoriasiform skin disease (Supplemental Figure 3 and data not shown). (A and B) A combination of $0.2 \mu \mathrm{g}$ murine rJE/MCP-1 and 5,000 U rTNF- $\alpha$, injected in $200 \mu \mathrm{l}$ PBS intradermally in the upper back skin of nonaffected CD18 hypo PL/J mice (A), led to the appearance of erythematous plaques covered with scales and crusts starting at day 4 after administration (B). (C and D) Skin samples from paraffin sections were stained with H\&E before (C) and 10 days after treatment, when epidermal hyperplasia and an abundant inflammatory infiltrate were observed within the dermis (D). (E and F) Cryosections from skin samples before (E) and after treatment stained for the keratinocyte marker K14-Alexa 488 (green) showed an increase in epidermal thickness and enhanced expression of the $\mathrm{K} 14$ proliferation-associated marker 10 days after treatment $(\mathbf{F})$. ( $\mathbf{G}$ and $\mathbf{H}$ ) At this same time point, a dermal inflammatory infiltrate predominantly consisting of $\mathrm{F} 4 / 80$-Alexa $488^{+}-$(green) and TNF- $\alpha-\mathrm{PE}^{+}-$activated (red) macrophages (overlay, yellow) was identified (H) compared with day $0(\mathbf{G})$. (I and $\mathbf{J})$ Interestingly, virtually no CD4-FITC+ T cells (green) were present in the dermis before (I) or after treatment with rMCP-1 and rTNF- $\alpha(\mathbf{J})$. Cell nuclei were counterstained with DAPI (blue). Dotted lines indicate the border between epidermis and dermis. Original magnification, $\times 20$.

$\mathrm{T}$ cells cannot (25). This may support the view that $\mathrm{T}$ cell emigration precedes the recruitment and activation of macrophages in the CD18 hypo murine model for chronic psoriasiform skin inflammation. This is further supported by the finding that adoptive transfer of effector/memory $\mathrm{T}$ cells $\left(\mathrm{CD} 4{ }^{+} \mathrm{CD} 62 \mathrm{~L}^{\text {low }} \mathrm{CD} 45 \mathrm{RB}{ }^{\text {low }}\right)$ derived from $C D 18$ hypo mice with a psoriasiform skin disease into the tail veins of healthy $C D 18^{\text {hypo }} \mathrm{PL} / \mathrm{J}$ mice resulted in the induction of the psoriasiform skin disease beginning at the ears and the facial area. Sequential skin biopsies revealed that at day 3 after the adoptive transfer $\mathrm{CD} 4^{+} \mathrm{T}$ cells were exclusively found, while at day 10 activated macrophages had also accumulated in high numbers (our unpublished observations).

Taken together, our results lead us to suggest a sequence of events where effector/memory $\mathrm{CD} 4^{+} \mathrm{T}$ cells $\left(\mathrm{CLA}^{+} \mathrm{CD} 44^{\text {high }} \mathrm{CD} 6\right.$ $\left.2 \mathrm{~L}^{\text {low }} \mathrm{CD} 45 \mathrm{RB}^{\text {low }}\right)$, upon activation, may contribute to the recruitment and activation of macrophages that produce proinflammatory cytokines such as TNF- $\alpha$, leading to the amplification of the inflammatory process and the full development of the psoriasiform inflammatory skin disease in PL/J CD18hypo mice. This view on effector/memory $\mathrm{T}$ cells in the immunopathogenesis of human psoriasis is shared by several reports $(18,56)$.

In the mouse model described by Stratis et al. (55), macrophage recruitment and activation depends on signals originating from inhibitor of NF-KB kinase 2-deficient (IKK2-deficient) keratinocytes, but not on the presence of $\mathrm{T}$ cells. The comparison of these 2 mouse models shows that in both $\mathrm{T}$ cell-independent and $\mathrm{T}$ cell-mediated psoriasis-like inflammatory skin disease, macrophages have pathogenic functions. It is possible that in the model described by Stratis et al. (55), IKK2-deficient keratinocytes can substitute for $\mathrm{T}$ cell signals and could recruit and activate macrophages, while in the CD18 hypo $\mathrm{PL} / \mathrm{J}$ model $\mathrm{T}$ cells are required for macrophage activation. Hence, the murine model described by Stratis et al. (55) would be more downstream in the pathogenic sequence of events compared with our model, with both resulting in severe psoriasis-like disease. Heterogeneity in the pathogenesis of psoriasis-like skin diseases in mice is also suggested by the existence of several $\mathrm{T}$ cell-dependent rodent models mimicking 
human psoriasis (11-13), in addition to a number of epidermisdependent murine models for psoriasis $(11,14,41,55,57)$. The understanding that complex clinical pictures may arise from dysregulations at different steps within the same pathogenic sequence of events may also have a general relevance for human psoriasis or other complex inflammatory diseases. In fact, human psoriasis is highly variable in its clinical picture. It is possible that psoriasis is also pathogenetically not a homogeneous entity, and while some cases may be caused by $\mathrm{T}$ cell-dependent activation of macrophages, others may occur via $\mathrm{T}$ cell-independent mechanisms of macrophage activation (58). These macrophage activation signals may be different and include antigen-specific activation of memory $\mathrm{T}$ cells, polyclonal activation of $\mathrm{T}$ cells by superantigens, and injury of the epidermis resulting in the release of proinflammatory cytokines and chemokines, a mechanism clinically known as the Koebner phenomenon in human psoriasis (59).

In conclusion, the PL/J CD18ypo mouse model demonstrates that the psoriasiform inflammatory skin disorder critically depends on efficient activation of macrophages with sufficient release of TNF- $\alpha$. Here, $\mathrm{CD}^{+} \mathrm{T}$ cells or simultaneous injection of MCP-1/TNF- $\alpha$ may have a distinct macrophage-activating role. This, in addition to other features shared with human psoriasis, makes this model a valuable tool for future investigations into the pathogenesis of chronic inflammatory skin diseases such as human psoriasis, including their polygenic base, and for future preclinical studies.

\section{Methods}

Mice. Mice with a hypomorphic mutation of the CD18 gene (CD18hypo in the $\mathrm{PL} / \mathrm{J}$ inbred strain were genotyped by Southern blot (15). CD18WT littermates resulting from heterozygote crosses served as WT controls. For the depletion of macrophages, neutralization of TNF- $\alpha$, and measurement of mRNA and protein levels of MCP-1, affected CD18 hypo mice with a strong psoriasiform phenotype were used. For the injection with rJE/MCP-1, LPS, rTNF- $\alpha$, or the combination of rJE/MCP- 1 and rTNF- $\alpha$, healthy $C D 18^{\text {hypo }}$ mice that did not show any psoriasiform phenotype were used. All mice were kept under specific pathogen-free conditions and were used for experiments at an age older than 6 weeks. Unless stated otherwise, skin tissue for cryosections was obtained from the neck area of mice. Subcutaneous injections were also performed in this location. All experiments were reviewed and approved by the Regierungspräsidium Tübingen, Tübingen, Germany, and performed in compliance with the German Law for Welfare of Laboratory Animals.

In vivo depletion of macrophages. Macrophages were depleted in vivo by using dichloromethylene diphosphonate (clodronate) encapsulated in liposomes. Clodronate liposomes and control liposomes were prepared as described previously (60). Clodronate was a gift of Roche Diagnostics. Mice were anesthetized with $200 \mu \mathrm{l}$ of narcotics (Ketanest S, $7 \mathrm{mg} / \mathrm{ml}$; Rompun, $1.2 \mathrm{~g} / \mathrm{ml}$; diluted in $0.9 \% \mathrm{NaCl}$ ), and $50 \mathrm{mg} / 200 \mu \mathrm{l}$ clodronate liposomes or control liposomes were injected subcutaneously at 4 sites $(50 \mu \mathrm{l} /$ site $)$ into lesional skin on the back of each mouse. Repeated injections of $200 \mu$ l clodronate liposomes or control liposomes were performed every 7 days for a period of 40 days. Macrophage depletion using clodronate liposomes was confirmed by staining skin cryosections with Alexa Fluor 488-conjugated Rat AntiMouse F4/80 mAb (clone CI: A3-1, CALTAG Laboratories; Invitrogen) or cervical lymph nodes cryosections with MOMA-2 (clone MOMA-2; Serotec). Before and after treatment, disease severity was determined by assessment of the clinical picture using an adapted PASI score as previously described (21). The specificity of macrophage depletion by liposome-encapsulated clodronate was extensively shown in the literature $(36-39,43,60)$ and confirmed for skin in this model by our own data (Supplemental Figure 1).
We found identical numbers of mature Langerin ${ }^{+} \mathrm{CD} 205^{+}$Langerhans/ dendritic cells in the chronic skin inflammation after treatment with clodronate liposomes compared with PBS control liposome treatment (Supplemental Figure 1). As immature dendritic cells may phagocytose clodronate liposomes, we cannot rule out the possibility that clodronate removes these immature Langerhans/dendritic cell populations.

Immunofluorescence staining. Frozen cryosections of skin and skin DLNs from mice of each genotype were fixed in ice-cold acetone for 10 minutes before staining. To detect murine macrophages, we used Alexa Fluor 488-conjugated Rat Anti-Mouse F4/80 (clone CI: A3-1, CALTAG Laboratories; Invitrogen) for the detection of mature tissue macrophages, and MOMA-2 (clone MOMA-2; Serotec) for the specific detection of the less mature monocyte/macrophage population in lymph nodes, as described previously (61). In addition, FITC-conjugated Rat Anti-Mouse CD4 (clone YTS191.1; Serotec), anti-mouse TNF- $\alpha$ (clone MP6-XT22; BD Biosciences - Pharmingen), rat anti-mouse Dectin 1 IgG2a (clone 218820; R\&D Systems), anti-iNOS polyclonal Ab (rabbit polyclonal Ab IgG; Affinity BioReagents), rabbit anti-mouse Arginase 1 (BD Biosciences - Pharmingen) in conjunction with streptavidin cytochrome 3 (Cy3) conjugate (Dianova Inc.) $\mathrm{mAb}$, and PE-conjugated anti-mouse Ly-6G and Ly-6C (GR-1; clone RB6-8C5; BD Biosciences - Pharmingen) were used. Propidium iodide (BD Biosciences - Pharmingen) and DAPI (Sigma-Aldrich) were used for staining of nuclei. All antibodies were diluted in a $1 \%$ antibody diluent (Dako). Immunohistochemistry was performed using a previously described protocol (13). Immunostainings were analyzed with a fluorescence microscope (Zeiss Axioskop 2 plus). Microscopic evaluation was performed by counting cells in the skin as previously described (21).

Administration of etanercept. Etanercept $(100 \mu \mathrm{g} /$ mouse $)$ was administrated i.p. every day for a period of 30 days to neutralize TNF- $\alpha$. In parallel, injection of $0.9 \% \mathrm{NaCl}$ was used as control. Effects of etanercept on the severity of the psoriasiform dermatitis were assessed by the adapted PASI score and immunostaining of skin cryosections as indicated above.

FACS analysis. The following mAbs were used for flow cytometry: MOMA-2 (clone MOMA-2; Serotec) and PE-conjugated anti-mouse I-A $\mathrm{A}^{\mathrm{b}}$ (clone AF6120.1; BD Biosciences - Pharmingen). Cervical LN cells isolated from either affected $C D 18^{\text {hypo }}$ or $C D 18^{W T} \mathrm{PL} / \mathrm{J}$ mice were processed as previously described $(21,25)$ and washed 2 times in $1 \times$ CellWASH (BD). For each staining, $10^{6}$ cells were incubated with $\mathrm{mAb}$ for 20 minutes at $4^{\circ} \mathrm{C}$, rinsed with PBS, and analyzed by flow cytometry (FACScan; BD).

RT-PCR. Lesional skin of affected CD18hypo mice or normal skin of CD18WT mice was excised and immediately frozen in liquid nitrogen, and total RNA was extracted using peqGOLD TriFast (peqLab), followed by DNase treatment. The contamination of genomic DNA was checked using specific MCP-1 primers as previously described (62). cDNA was prepared from these samples using First Strand cDNA Synthesis Kit for RT-PCR (AMV)+ (Roche Diagnostics), and Oligo- $\mathrm{p}(\mathrm{dT})_{15}$ primers were used for reverse transcription according to manufacturer's instructions. A capillary realtime thermocycler (LightCycler; Roche Diagnostics) was used to perform quantitative PCR as described previously (62). cDNA derived from lesional skin of 3 additional affected $C D 18^{\text {hypo }}$ mice or normal skin of $C D 18^{W T}$ mice was pooled to perform semiquantitative RT-PCR. The PCR amplification consisted of 30 cycles of 5 minutes at $95^{\circ} \mathrm{C}, 30$ seconds at $60^{\circ} \mathrm{C}$, and $30 \mathrm{sec}-$ onds at $72^{\circ} \mathrm{C}$ followed by 1 cycle at $72^{\circ} \mathrm{C}$ for 5 minutes. The PCR products were visualized by ethidium bromide in $1 \%$ agarose gels.

ELISA. Affected CD18hypo mouse lesional skin or CD18WT mouse normal skin samples were rinsed in ice-cold PBS and snap-frozen in liquid nitrogen. Frozen samples were ground using a Micro-Dismembrator (Biotech International Inc.) and resuspended in homogenization RIPA buffer (SigmaAldrich) at $100 \mathrm{mg} / \mathrm{ml}$. The homogenate was centrifuged at $20,800 \mathrm{~g}$ for 20 minutes at $4^{\circ} \mathrm{C}$. The supernatants were collected and stored at $-80^{\circ} \mathrm{C}$ until 
ELISA was performed. Mouse specific MCP-1 ELISA kit (Pierce; Endogen) was used according to the manufacturer's instruction to measure MCP-1 protein levels. MCP-1 values were standardized to milligram of tissue.

Simultaneous intradermal injection of murine $r J E / M C P-1$ and murine $r T N F-\alpha$. A combination of $0.2 \mu \mathrm{g}$ mouse rJE/MCP-1 (R\&D Systems) and 5,000 U mouse rTNF- $\alpha$ (Promokine) diluted in $200 \mu \mathrm{l}$ PBS was injected intradermally at 4 sites $(50 \mu \mathrm{l} /$ site $)$ on the back of each healthy $C D 18^{\text {hypo }}$ mouse. As controls, $200 \mu \mathrm{l}$ of PBS and rJE/MCP-1 as well as rTNF- $\alpha$ as single substances were injected. Injected skin areas were excised at defined time points ( 24 hours, 4 days, and 10 days) after treatment, cryopreserved, and subsequently analyzed by means of $\mathrm{H} \& \mathrm{E}$ and immunofluorescent staining using antibodies directed against the keratinocyte marker keratin 14 (K14), the macrophage marker F4/80, the T cell marker CD4, and TNF- $\alpha$. Immunostainings for keratinocytes, activated TNF- $\alpha$-producing macrophages, and $\mathrm{CD} 4^{+} \mathrm{T}$ cells were analyzed with a fluorescence microscope (Zeiss Axioskop 2 FS plus).

When the $C D 18^{\text {hypo }}$ mutation was backcrossed onto the $\mathrm{PL} / \mathrm{J}$ inbred strain, virtually all homozygous mice developed a chronic inflammatory skin disease with a mean age of onset of 11 weeks after birth (16). We used CD18hypo $\mathrm{PL} / \mathrm{J}$ mice for the injection studies with rMCP-1, LPS, rTNF- $\alpha$, or the combination of rMCP- 1 and rTNF- $\alpha$ at earlier time points, where the manifestation of the spontaneous chronic skin inflammation had not yet occurred.

Statistics. Quantitative results are presented as means \pm SD. Mean values were tested by means of a 2-tailed heteroscedastic Student's $t$ test, or in cases of a non-Gaussian distribution, Mann-Whitney $U$ test was used. Differences were considered to be statistically significant at $P<0.05$ and $P<0.01$.

\section{Acknowledgments}

We are grateful to Britta Bartelt-Kirbach (University of Ulm) for technical assistance of RT-PCR. We would like to thank Robert Blakytny for critical discussion and reading of the manuscript. This work was supported by the German Research Foundation (DFG) within the SFB 497 "Signals and Signal Processing during Cellular Differentiation," and the individual DFG research grants SU 195/3-1 (to C. Sunderkötter) and SCHA 411/12-1 (to K. Scharffetter-Kochanek).

Received for publication October 20, 2005, and accepted in revised form May 30, 2006.

Address correspondence to: Karin Scharffetter-Kochanek, Department of Dermatology and Allergic Diseases, University of Ulm, Maienweg 12, D-89081 Ulm, Germany. Phone: 49-731-500-21801; Fax: 49-731-500-21870; E-mail: karin.scharffetter-kochanek@ uniklinik-ulm.de.

Honglin Wang and Thorsten Peters contributed equally to this work.
1. Nickoloff, B.J. 1999. The immunologic and genetic basis of psoriasis. Arch. Dermatol. 135:1104-1110.

2. Nickoloff, B.J., and Nestle, F.O. 2004. Recent insights into the immunopathogenesis of psoriasis provide new therapeutic opportunities. J. Clin. Invest. 113:1664-1675. doi:10.1172/JCI200422147.

3. Gillitzer, R., et al. 1993. MCP-1 mRNA expression in basal keratinocytes of psoriatic lesions. J. Invest. Dermatol. 101:127-131.

4. van den Oord, J.J., and de Wolf-Peeters, C. 1994. Epithelium-lining macrophages in psoriasis. $\mathrm{Br}$. J. Dermatol. 130:589-594.

5. Djemadji-Oudjiel, N., Goerdt, S., Kodelja, V., Schmuth, M., and Orfanos, C.E. 1996. Immunohistochemical identification of type II alternatively activated dendritic macrophages (RM 3/1+3, MS-1+/-, 25F9-) in psoriatic dermis. Arch. Dermatol. Res. 288:757-764.

6. Antoni, C., and Manger, B. 2002. Infliximab for psoriasis and psoriatic arthritis. Clin. Exp. Rheumatol. 20(6 Suppl. 28):S122-S125.

7. Wakefield, P.E., James, W.D., Samlaska, C.P., and Meltzer, M.S. 1991. Tumor necrosis factor. J. Am. Acad. Dermatol. 24:675-685.

8. Nickoloff, B.J. 1991. The cytokine network in psoriasis. Arch. Dermatol. 127:871-884.

9. Leonardi, C.L., et al. 2003. Etanercept as monotherapy in patients with psoriasis. N. Engl. J. Med. 349:2014-2022.

10. Gottlieb, A.B., et al. 2005. TNF inhibition rapidly down-regulates multiple proinflammatory pathways in psoriasis plaques. J. Immunol. 175:2721-2729.

11. Sano, S., et al. 2005. Stat3 links activated keratinocytes and immunocytes required for development of psoriasis in a novel transgenic mouse model. Nat. Med. 11:43-49.

12. Schon, M.P., Detmar, M., and Parker, C.M. 1997. Murine psoriasis-like disorder induced by naive CD4+ T cells. Nat. Med. 3:183-188.

13. Pasparakis, M., et al. 2002. TNF-mediated inflammatory skin disease in mice with epidermis-specific deletion of IKK2. Nature. 417:861-866.

14. Zenz, R., et al. 2005. Psoriasis-like skin disease and arthritis caused by inducible epidermal deletion of Jun proteins. Nature. 437:369-375.

15. Wilson, R.W., et al. 1993. Gene targeting yields a CD18-mutant mouse for study of inflammation.
J. Immunol. 151:1571-1578.

16. Bullard, D.C., et al. 1996. A polygenic mouse model of psoriasiform skin disease in CD18-deficient mice. Proc. Natl. Acad. Sci. U. S. A. 93:2116-2121.

17. Shuster, S. 1972. Psoriatic alopecia. Br. J. Dermatol. 87:73-77.

18. Schon, M.P., and Boehncke, W.H. 2005. Psoriasis. N. Engl. J. Med. 352:1899-1912.

19. Feldman, S.R., et al. 2001. Betamethasone valerate in foam vehicle is effective with both daily and twice a day dosing: a single-blind, open-label study in the treatment of scalp psoriasis. J. Cutan. Med. Surg. 5:386-389.

20. van de Kerkhof, P.C., and Franssen, M.E. 2001. Psoriasis of the scalp. Diagnosis and management. Am. J. Clin. Dermatol. 2:159-165.

21. Kess, D., et al. 2003. CD4+ T cell-associated pathophysiology critically depends on CD18 gene dose effects in a murine model of psoriasis. J. Immunol. 171:5697-5706.

22. Morel, P., et al. 1992. Anti-CD4 monoclonal antibody therapy in severe psoriasis. J. Autoimmun. 5:465-477.

23. Hynes, R.O. 1987. Integrins: a family of cell surface receptors. Cell. 48:549-554.

24. Carlos, T.M., and Harlan, J.M. 1994. Leukocyteendothelial adhesion molecules. Blood. 84:2068-2101.

25. Grabbe, S., et al. 2002. $\beta 2$ integrins are required for skin homing of primed $\mathrm{T}$ cells but not for priming naive T cells. J. Clin. Invest. 109:183-192. doi:10.1172/JCI200211703.

26. Fine, J.S., and Kruisbeek, A.M. 1991. The role of LFA-1/ICAM-1 interactions during murine T lymphocyte development. J. Immunol. 147:2852-2859.

27. Quddus, J., Kaplan, A., and Richardson, B.C. 1994. Anti-CD11a prevents deletion of self-reactive T cells in neonatal C57BR mice. Immunology. 82:301-305.

28. van de Kerkhof, P.C., and Weemaes, C.M. 1990. Skin manifestations in congenital deficiency of leucocyte-adherence glycoproteins (CDLG). Br. J. Dermatol. 123:395-401.

29. Tomfohrde, J., et al. 1994. Gene for familial psoriasis susceptibility mapped to the distal end of human chromosome 17q. Science. 264:1141-1145.

30. Gencik, M., et al. 2000. The association of CD18 alleles with anti-myeloperoxidase subtypes of ANCA-associated systemic vasculitides. Clin.
Immunol. 94:9-12.

31. Meller, S., et al. 2001. Novel SNPs in the CD18 gene validate the association with MPO-ANCA+ vasculitis. Genes Immun. 2:269-272.

32. Elliott, M.J., et al. 1994. Randomised double-blind comparison of chimeric monoclonal antibody to tumour necrosis factor alpha (cA2) versus placebo in rheumatoid arthritis. Lancet. 344:1105-1110.

33. van Roon, J.A., Lafeber, F.P., and Bijlsma, J.W. 2001. Synergistic activity of interleukin-4 and interleukin-10 in suppression of inflammation and joint destruction in rheumatoid arthritis. Arthritis Rheum. 44:3-12.

34. Thepen, T., et al. 2000. Resolution of cutaneous inflammation after local elimination of macrophages. Nat. Biotechnol. 18:48-51.

35. Kurimoto, I., van Rooijen, N., Dijkstra, C.D., and Streilein, J.W. 1994. Role of phagocytic macrophages in induction of contact hypersensitivity and tolerance by hapten applied to normal and ultraviolet B-irradiated skin. Immunology. 83:281-287.

36. Van Rooijen, N., Kors, N., vd Ende, M., and Dijkstra, C.D. 1990. Depletion and repopulation of macrophages in spleen and liver of rat after intravenous treatment with liposome-encapsulated dichloromethylene diphosphonate. Cell Tissue Res. 260:215-222.

37. Van Rooijen, N., and van Kesteren-Hendrikx, E. 2003. "In vivo" depletion of macrophages by liposome-mediated "suicide". Methods Enzymol. 373:3-16.

38. Sunderkötter, C., et al. 2004. Subpopulations of mouse blood monocytes differ in maturation stage and inflammatory response. J. Immunol. 172:4410-4417.

39. Dupasquier, M., Stoitzner, P., van Oudenaren, A., Romani, N., and Leenen, P.J. 2004. Macrophages and dendritic cells constitute a major subpopulation of cells in the mouse dermis. J. Invest. Dermatol. 123:876-879.

40. Lu, B., et al. 1998. Abnormalities in monocyte recruitment and cytokine expression in monocyte chemoattractant protein 1-deficient mice. J. Exp. Med. 187:601-608.

41. Nickoloff, B.J. 2000. Characterization of lymphocyte-dependent angiogenesis using a SCID mouse: human skin model of psoriasis. J. Investig. Dermatol. 
Symp. Proc. 5:67-73.

42. Vestergaard, C., et al. 2004. Expression of CCR2 on monocytes and macrophages in chronically inflamed skin in atopic dermatitis and psoriasis. Acta Derm. Venereol. 84:353-358.

43. Drevets, D.A., et al. 2004. The Ly-6Chigh monocyte subpopulation transports Listeria monocytogenes into the brain during systemic infection of mice. J. Immunol. 172:4418-4424.

44. Grewal, I.S., et al. 1997. Transgenic monocyte chemoattractant protein-1 (MCP-1) in pancreatic islets produces monocyte-rich insulitis without diabetes: abrogation by a second transgene expressing systemic MCP-1. J. Immunol. 159:401-408.

45. Nakamura, K., Williams, I.R., and Kupper, T.S. 1995. Keratinocyte-derived monocyte chemoattractant protein 1 (MCP-1): analysis in a transgenic model demonstrates MCP-1 can recruit dendritic and Langerhans cells to skin. J. Invest. Dermatol. 105:635-643.

46. Stout, R.D., and Suttles, J. 1993. T cell-macrophage cognate interaction in the activation of macrophage effector function by Th2 cells. J. Immunol. 150:5330-5337.

47. Schlaak, J.F., et al. 1994. T cells involved in psoriasis vulgaris belong to the Th1 subset. J. Invest. Dermatol. 102:145-149.

48. Ackermann, L., and Harvima, I.T. 1998. Mast cells of psoriatic and atopic dermatitis skin are positive for TNF-alpha and their degranulation is associated with expression of ICAM- 1 in the epidermis. Arch. Dermatol. Res. 290:353-359.

49. Xaus, J., et al. 2000. LPS induces apoptosis in macrophages mostly through the autocrine production of TNF-alpha. Blood. 95:3823-3831.

50. Ghoreschi, K., et al. 2003. Interleukin-4 therapy of psoriasis induces Th2 responses and improves human autoimmune disease. Nat. Med. 9:40-46.

51. Trepicchio, W.L., et al. 1999. Interleukin-11 therapy selectively downregulates type I cytokine proinflammatory pathways in psoriasis lesions. J. Clin. Invest. 104:1527-1537.

52. Kauffman, C.L., et al. 2004. A phase I study evaluating the safety, pharmacokinetics, and clinical response of a human IL-12 p40 antibody in subjects with plaque psoriasis. J. Invest. Dermatol. 123:1037-1044.

53. Palladino, M.A., Bahjat, F.R., Theodorakis, E.A., and Moldawer, L.L. 2003. Anti-TNF-alpha therapies: the next generation. Nat. Rev. Drug Discov. 2:736-746.

54. Ozawa, M., and Aiba, S. 2004. Immunopathogenesis of psoriasis. Curr. Drug Targets Inflamm. Allergy. 3:137-144.

55. Stratis, A., et al. 2006. Pathogenic role for skin macrophages in a mouse model of keratinocyte- induced psoriasis-like skin inflammation. J. Clin. Invest. 116:2094-2104. doi:10.1172/JCI27179.

56. Ellis, C.N., and Krueger, G.G. 2001. Treatment of chronic plaque psoriasis by selective targeting of memory effector T lymphocytes. $N$. Engl. J. Med. 345:248-255.

57. Li, A.G., Wang, D., Feng, X.H., and Wang, X.J. 2004. Latent TGFbeta1 overexpression in keratinocytes results in a severe psoriasis-like skin disorder. EMBO J. 23:1770-1781.

58. Nickoloff, B.J. 2006. Keratinocytes regain momentum as instigators of cutaneous inflammation. Trends Mol. Med. 12:102-106.

59. Miller, R.A. 1982. The Koebner phenomenon. Int. J. Dermatol. 21:192-197.

60. Van Rooijen, N., and Sanders, A. 1994. Liposome mediated depletion of macrophages: mechanism of action, preparation of liposomes and applications. J. Immunol. Methods. 174:83-93.

61. Kraal, G., Rep, M., and Janse, M. 1987. Macrophages in $\mathrm{T}$ and $\mathrm{B}$ cell compartments and other tissue macrophages recognized by monoclonal antibody MOMA-2. An immunohistochemical study. Scand. J. Immunol. 26:653-661.

62. Shinozaki, M., et al. 2002. IL-15, a survival factor for kidney epithelial cells, counteracts apoptosis and inflammation during nephritis. J. Clin. Invest. 109:951-960. doi:10.1172/JCI200214574. 